\title{
Nomenclature Committee of IUB (NC-IUB) and \\ IUB-IUPAC Joint Commission on Biochemical Nomenclature (JCBN)
}

\section{Newsletter 1983}

NC-IUB and JCBN hope that their Newsletters, designed to inform scientists about the work of the committees, may help the biochemical community. They are intended to call attention to features in recently published recommendations. Since we plan to prepare a new edition of Enzyme Nomenclature fairly soon, we particularly ask that suggestions on the listing of enzymes should reach NC-IUB by 1st April 1983.

The 1982 Newsletter was published in: Arch. Biochem. Biophys. (1982) 213, 734-736; Biochem. Int. (1982) 4, after p. 116; Eur. J. Biochem. (1982) 122, 437-438; Hoppe-Seyler's Z. Physiol. Chem. (1982) 363, I-IV; and a summary in Trends Biochem. Sci. (1982) 7, 10.

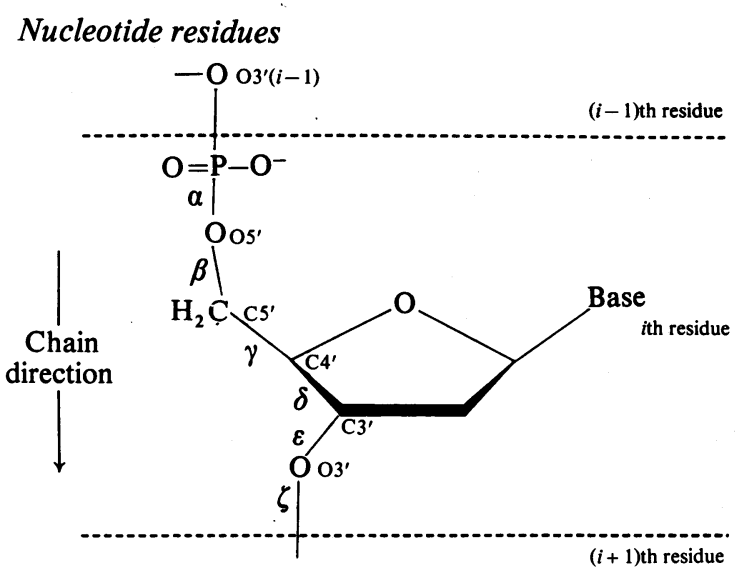

Monosaccharide residues

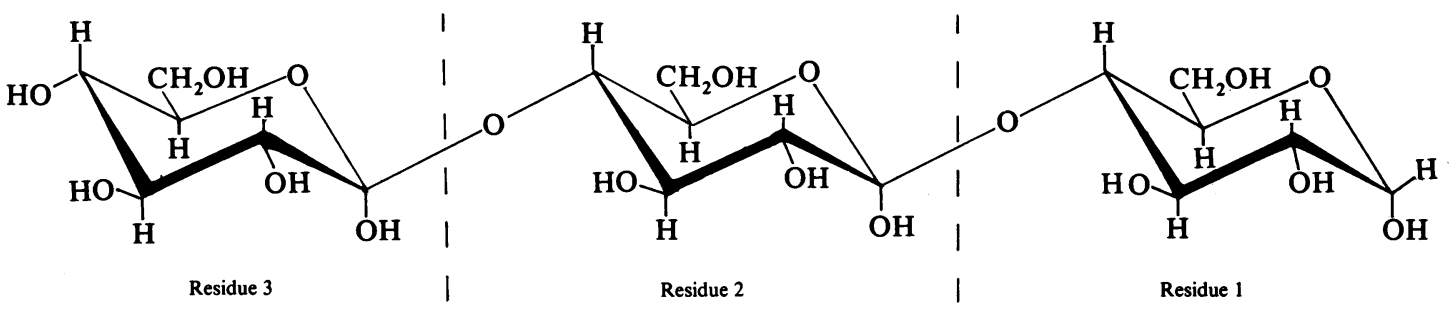

Residues in oligosaccharide chains are numbered as shown above, from the reducing end, in Symbols for Specifying the Conformation of Polysaccharide. Chains [1]. Although this has the inconvenience of numbering from right to left in the conventional representation of oligosaccharides, it has the advantage that shortening and lengthening of chains by the usual mechanism of transglycosylation at the nonreducing end leaves the numbering unaltered for the residues remaining in the chain.

The limits of residues are those indicated by the broken lines, according to the same recommendations. The bridging oxygen is thus part of the residue glycosylated, i.e. of the residue that contributes it in both chemical and biochemical synthesis. In shortened representations of oligosaccharide chains [2], e.g. Glc ( $\alpha 1-4)$ Glc, each three-letter symbol represents a residue, so that the bridging oxygen is included in the right-hand one of a pair of symbols. Since adding a hyphen to the right of the symbol for a sugar removes its anomeric hydroxyl, it is therefore necessary to represent explicitly the glycosylated oxygen of the aglycone when a glycoside is represented by symbols, e.g. $\operatorname{Man}(\alpha)$-OMe for methyl $\alpha$-D-mannoside.
The limits and numbering of nucleotide residues are as shown above in Abbreviations and Symbols for the Description of Conformations of Polynucleotide Chains [3]. Thus the $i$ th residue starts with its phosphorus atom, and runs through $\mathrm{O5}^{\prime}$, $\mathrm{C5}^{\prime}, \mathrm{C4}^{\prime}$ and $\mathrm{C3}^{\prime}$ to $\mathrm{O}^{\prime}$ ', which is attached to $\mathrm{P}$ of the $(i+1)$ th residue. The residue therefore corresponds with the group transferred from a single nucleoside triphosphate during biosynthesis, i.e. the residue of that molecule after removal of diphosphate (pyrophosphate).

The torsion angles are designated as shown, so that, for example, $\beta$ represents the torsion angle about the $\mathrm{O5}^{\prime}-\mathrm{C5}^{\prime}$ bond. This is defined (both in earlier IUPAC recommendations for organic chemistry in general and in the new recommendations for polynucleotides) as the angle through which the bond $\mathrm{P}-\mathrm{O5}^{\prime}$ needs to be rotated in a clockwise direction, when viewed along the $\mathrm{O}^{\prime}-\mathrm{C5}^{\prime}$ bond, in order to eclipse the $\mathrm{C}^{\prime}-\mathrm{C} 4$ ' bond.

\section{Nomenclature of vitamin $D$}

The new recommendations [4] propose an extended system of trivial and semisystematic names for 
important metabolites of vitamin D. The names cholecalciferol and ergocalciferol are retained as alternatives to calciol and ercalciol respectively. The trivial names calcidiol, calcitriol and calcitetrol are reserved for the 3,25-diol, the 1,3,25-triol and the $1,3,24,25$-tetrol respectively. The new trivial names tacalciol and isocalciol are proposed for tachysterol ${ }_{3}$ and isovitamin $D_{3}$ respectively.

For semisystematic names, the most important change from past practice is the recommendation that all stereochemistry in ring A or at C-6 or C-7 should use $R, S, E$ or $Z$. This prevents confusion arising about the meaning of $\alpha$ or $\beta$, as ring $A$ is free to turn over relative to rings $C$ and $D$.

\section{Retinoid nomenclature}

New recommendations [5] are based on three defined parent compounds: retinol, retinal and retinoic acid. The corresponding hydrocarbon is called deoxyretinol; the name retinene is unsuitable, both because this has been used in the past for retinal, and because it suggests a compound with only a single double bond.

\section{Symbolism and terminology in enzyme kinetics}

The new recommendations [6] attempt to describe and systematize existing practice and to make it consistent as far as possible with IUPAC's Symbolism and Terminology in Chemical Kinetics [7]. Although commonly used names for describing mechanisms are included, most of the definitions are operational rather than mechanistic, i.e. they define what is observed and not its interpretation.

The names 'maximum velocity' and 'maximum rate' for the extrapolated rate of an enzymecatalysed reaction saturated with substrate(s) are discouraged, because they do not refer to a maximum in the mathematical sense; the name limiting rate, symbol $V$, is recommended instead.
A in this example, is not fixed but defines the particular substrate considered.

The inhibition constant that defines the effect of an inhibitor on the apparent value of the specificity constant is called the competitive inhibition constant, symbol $K_{\mathrm{lc}}$, whereas the inhibition constant that defines the effect of an inhibitor on the apparent value of the catalytic constant is called the uncompetitive inhibition constant, symbol $K_{\mathrm{lu}}$. Either of these quantities may, however, be called the inhibition constant, symbol $K_{1}$, if care is taken to avoid ambiguity.

The recommendations set out to be as permissive as is consistent with clarity, and they include various alternative names and symbols not mentioned in this note. Such permissiveness places an extra obligation on authors and journals to avoid ambiguity.

\section{Enzyme nomenclature}

Supplemerits to Enzyme Nomenclature have appeared, the latest being Supplement 3 [Eur. $J$. Biochem. (1982) 125, 1-13] and Supplement 4 is in press. Changes and additions in these supplements are given below. Readers are reminded of the invitation to submit suggestions for additions and changes. If these are to be considered in time for the next edition, they should reach NG-IUB by 1 April 1983. i

Tetrapyrrole formation. EC 4.3.1.8 has been renamed in Supplement 3 as hydroxymethylbilane synthase, because its earlier name of uroporphyrinogen I synthase was based on a mistaken view of the reaction it catalyses. It is now known to catalyse the formation of hydroxymethylbilane and four molecules of ammonia from four molecules of porphobilinogen and one of water. The next step in porphyrin biosynthesis is the conversion of hydroxymethylbilane into a cyclic tetrapyrrole with reversal of ring $\mathrm{D}$. This is catalysed by uroporphyrinogen-III synthase, now listed as EC 4.2.1.75:<smiles>NCc1[nH]cc(Cl)c1CCl</smiles>

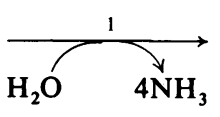

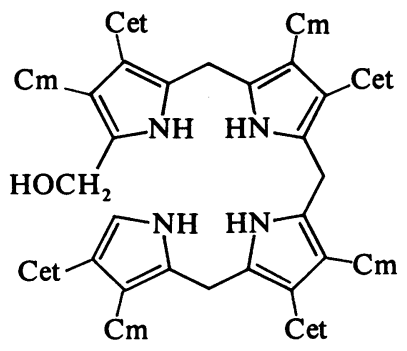

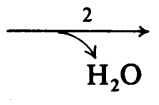

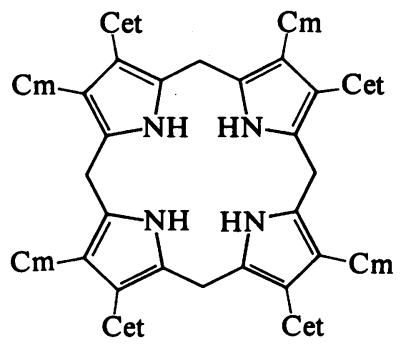

This quantity divided by the concentration of catalytic centres is called the catalytic constant, symbol $k_{0}$. Many authors have commented on the lack of a generally recognized name and symbol for the ratio $k_{0} / K_{\mathrm{m}}$ : the name specificity constant, symbol $k_{\mathrm{A}}$, is recommended, in which the subscript, where $-\mathrm{Cm}$ is carboxymethyl $\left(-\mathrm{CH}_{2}-\mathrm{COOH}\right)$, - Cet is 2-carboxyethyl $\left(-\left[\mathrm{CH}_{2}\right]_{2}-\mathrm{COOH}\right)$, reaction 1 is hydroxymethylbilane synthase (EC 4.3.1.8) and reaction 2 is uroporphyrinogen-III synthase (EC 4.2.1.75).

Aspartic and cysteine proteinases. The enzymes 
previously called thiol proteinases (EC 3.4.22) are now (Supplement 2) called cysteine proteinases; those previously called carboxyl (acid) proteinases (EC 3.4.23) are now called aspartic proteinases. This specification of the residue that can be acylated at the active site makes them analogous in naming to the serine proteinases (EC 3.4.21).

Replacement of $\mathrm{CHOH}$ by $\mathrm{CO}$. The compounds pantoate, $\mathrm{HOCH}_{2}-\mathrm{C}\left(\mathrm{CH}_{3}\right)_{2}-\mathrm{CHOH}-\mathrm{COO}^{-}$, and sphinganine, $\quad \mathrm{CH}_{3}-\left[\mathrm{CH}_{2}\right]_{14}-\mathrm{CHOH}-\mathrm{CH}\left(\mathrm{NH}_{2}\right)-$ $\mathrm{CH}_{2} \mathrm{OH}$, are both biosynthesized from the corresponding compounds in which $\mathrm{CO}$ replaces $\mathrm{CHOH}$. Use of the prefix oxo, which replaces $\mathrm{CH}_{2}$ with $\mathrm{CO}$, would give cumbersome names for such compounds, because it would have to follow the prefix deoxy, which replaces $\mathrm{CHOH}$ with $\mathrm{CH}_{2}$. Hence we use the prefix dehydro to replace $\mathrm{CHOH}$ with $\mathrm{CO}$, as already well established with shikimate and quinate. This is used for dehydropantoate hydroxymethyltransferase (EC 2.1.2.11), newly listed in Supplement 3 , and in re-writing the reaction of serine palmitoyltransferase (EC 2.3.1.50) to show that its product is 3-dehydro-D-sphinganine.

Teichoic acid biosynthesis. CDPglycerol glycerolphosphotransferase (EC 2.7.8.12) is responsible for the transfer of $-\mathrm{P}-\mathrm{O}-[\mathrm{CHOH}]_{n}-\mathrm{H}$ groups where $n=3$ in the biosynthesis of one teichoic acid. When $n=5$ a different teichoic acid is made, and the enzyme responsible, CDPribitol ribitolphosphotransferase (EC 2.7.8.14), is now listed in Supplement 3.

Polyamine synthesis. The enzyme that transfers a 3-aminopropyl group onto putrescine (butane-1,4diamine) to form spermidine is now (Supplement 3) called spermidine synthase (EC 2.5.1.16) instead of aminopropyltransferase, to distinguish it from spermine synthase (EC 2.5.1.22), which was not previously listed and which also acts by transfer of the aminopropyl group. Similarly sym-norspermidine synthase (EC 2.5.1.23 in Supplement 4) transfers the 2-aminopropyl group onto propane1,3-diamine to form sym-norspermidine, i.e. bis(3-aminopropyl)amine.

Residues in proteins as enzyme substrates. Hitherto enzymes that act on amino-acid residues in proteins have been named in different ways. For some of them, there has been no indication that the substrate is not a free amino acid, e.g. lysine, 2-oxoglutarate dioxygenase (EC 1.14.11.4); for some, 'peptidyl-' has been inserted before the name of the amino acid, e.g. peptidyl-lysine oxidase (EC 1.4.3.13), rather suggesting a $C$-terminal residue; for some, the reverse practice, e.g. glutaminyl-peptide $\gamma$-glutamyltransferase (EC 2.3.1.13), suggests an $\mathrm{N}$-terminal residue; a fourth practice is seen in the name protein (arginine) methyltransferase (EC 2.1.1.23). Sometimes biochemists have changed the name of the amino acid by replacing 'ine' with 'yl', but this indicates an acyl group rather than an amino-acid residue (thus an internal lysine residue is an acylated lysyl group), so this is not done in Enzyme Nomenclature. We plan to standardize by adding 'protein-', or the name of a particular protein, to the name of the residue that is the substrate. This has been done in Supplement 4, e.g.:

EC 1.4.3.13 protein-lysine 6-oxidase

EC 1.14.11.4 procollagen-lysine, 2-oxoglutarate 5-dioxygenase

EC 2.1.1.23 protein-arginine methyltransferase

EC 2.1.1.24 protein-glutamic(aspartic) methyltransferase

EC 2.1.1.43 histone-lysine methyltransferase EC 2.1.1.60 calmodulin-lysine methyltransferase

EC 2.3.2.13 protein-glutamine glutaminase

The group $-\mathrm{C}(\mathrm{COOH})=\mathrm{CH}_{2}$ in chorismate and UDPmuramate synthesis. The 1-carboxyvinyl group has been given various names based on pyruvate in the past. These are misleading because they imply the presence of oxygen on the central carbon atom, and because they suggest that the group is an acyl group. By contrast, the carboxymethyl group is not named as a derivative of acetate. NC-IUB therefore, despite its great reluctance to change existing names, believes that it will be much less confusing to give this group its systematic name of 1-carboxyvinyl. This leads to revised entries for four enzymes in Supplement 4:

EC 1.1.1.158 UDPacetylmuramate dehydrogenase

EC 2.5.1.7 UDPacetylglucosamine 1-carboxyvinyltransferase

EC 2.5.1.19 3-phosphoshikimate 1-carboxyvinyltransferase

EC 4.6.1.4 chorismate synthase

Oxidation products of cysteine and methionine. The groups $-\mathrm{SO}_{3} \mathrm{H},-\mathrm{SO}_{2} \mathrm{H},-\mathrm{SOH},-\mathrm{SO}_{2}-$ and -SO- are specified by the suffixes sulphonic acid, sulphinic acid, sulphenic acid, sulphone and sulphoxide. In this nomenclature, the rest of the molecule is separately specified, e.g. benzenesulphonic acid, $\mathrm{Ph}-\mathrm{SO}_{3} \mathrm{H}$, dimethyl sulphoxide, $\mathrm{Me}_{2} \mathrm{SO}$. Each name therefore specifies the presence of sulphur only in the suffix, so that it is wrong to add such a suffix to the name of a compound that already contains sulphur in order to indicate a change in the oxidation state of that sulphur. Hence using a name such as 'cysteine sulphinic acid' suggests addition of $-\mathrm{SO}_{2} \mathrm{H}$ to cysteine, rather than oxidation of the thiol group; such misuse of terms is particularly likely to cause confusion with cysteine derivatives in view of the fact that both cysteic acid, whose side chain is $-\mathrm{CH}_{2}-\mathrm{SO}_{3} \mathrm{H}$, and $\mathrm{S}$-sulphocysteine, whose side chain is $-\mathrm{CH}_{2}-\mathrm{S}-\mathrm{SO}_{3} \mathrm{H}$, feature in protein chemistry. The oxidized forms of cysteine may be called 
cysteic acid, 3-sulphinoalanine and 3-sulphenoalanine, and those of methionine may be called methionine $S S$-dioxide and methionine $S$-oxide. In conformity with this, the reaction of cysteine dioxygenase (EC 1.13.11.20) has been re-written in Supplement 4 to show that its product is 3sulphinoalanine, and the enzyme that decarboxylates this to yield hypotaurine is now called 3-sulphinoalanine decarboxylase (EC 4.1.1.29).

\section{Personnel}

P. Karlson retired from the chairmanship of the committees on 31 December 1982, and became an associate member. The new chairman is H. B. F. Dixon, who is succeeded as secretary by $A$. Cornish-Bowden (Department of Biochemistry, University of Birmingham, P.O. Box 363, Birmingham B15 2TT, U.K.) to whom suggestions and comments on nomenclature recommendations should be sent.

1. IUPAC-IUB Joint Commission on Biochemical Nomenclature (JCBN) Symbols for Specifying the Conformation of Polysaccharide Chains, Recommendations 1981: Eur.J. Biochem. (1983), in the press
2. IUPAC-IUB Joint Commission on Biochemical Nomenclature (JCBN) Abbreviated Terminology of Oligosaccharide Chains, Recommendations 1980: J. Biol. Chem. (1982) 257, 3347-3351; Eur. J. Biochem. (1982) 126, 433-437; Pure Appl. Chem. (1982) 54, $1517-1522$

3. IUPAC-IUB Joint Commission on Biochemical Nomenclature (JCBN) Abbreviations and Symbols for the Description of Conformations of Polynucleotide Chains, Recommendations 1982: Eur. J. Biochem. (1983), in the press

4. IUPAC-IUB Joint Commission on Biochemical Nomenclature (JCBN) Nomenclature of Vitamin D, Recommendations 1981: Eur. J. Biochem. (1982) 124, 223-227; Pure Appl. Chem. (1982) 54, 1511-1516

5. IUPAC-IUB Joint Commission on Biochemical Nomenclature (JCBN) Nomenclature of Retinoids, Recommendations 1981: Eur. J. Biochem. (1982) 129, $1-5$

6. Nomenclature Committee of the International Union of Biochemistry (NC-IUB) Symbolism and Terminology in Enzyme Kinetics, Recommendations 1981: Eur. J. Biochem. (1982) 128, 281-291

7. IUPAC Physical Chemistry Division Symbolism and Terminology in Chemical Kinetics: Pure Appl. Chem. (1981) 53, 753-771 